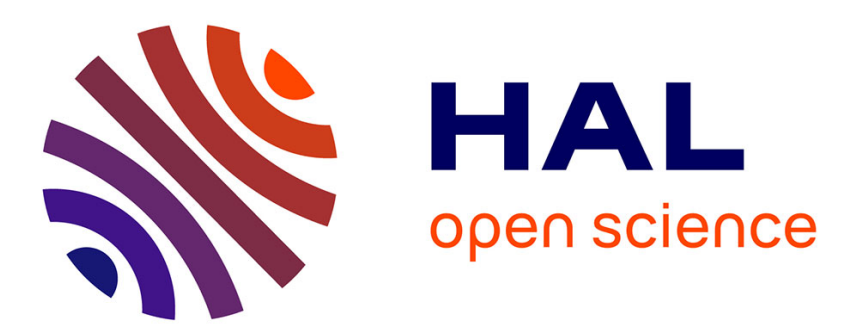

\title{
Possibilité d'utilisation des légumineuses arborescentes en forêt tempérée
}

\author{
Frédéric Vernier, François Le Tacon
}

\section{To cite this version:}

Frédéric Vernier, François Le Tacon. Possibilité d'utilisation des légumineuses arborescentes en forêt tempérée. Revue forestière française, 1988, 60 (3), pp.213-223. 10.4267/2042/25885 . hal-03424624

\section{HAL Id: hal-03424624 \\ https://hal.science/hal-03424624}

Submitted on 10 Nov 2021

HAL is a multi-disciplinary open access archive for the deposit and dissemination of scientific research documents, whether they are published or not. The documents may come from teaching and research institutions in France or abroad, or from public or private research centers.
L'archive ouverte pluridisciplinaire HAL, est destinée au dépôt et à la diffusion de documents scientifiques de niveau recherche, publiés ou non, émanant des établissements d'enseignement et de recherche français ou étrangers, des laboratoires publics ou privés. 


\title{
biologie et forêt
}

\section{POSSIBILITÉ D'UTILISATION DES LÉGUMINEUSES ARBORESCENTES EN FORÊT TEMPÉRÉE}

\author{
F. VERNIER - F. LE TACON
}

L'azote minéral est un des éléments clés de la production végétale. Sa disponibilité est souvent insuffisante dans les sols des forêts tempérées. La plupart des essais de fertilisation effectués sur peuplements adultes montre en effet une réponse à l'apport de matière azotée (Hausser, 1961 ; Viro, 1969 ; Bonneau, 1974 ; Nys, 1984).

Pour améliorer la disponibilité des sols forestiers en azote, plusieurs solutions sont envisageables.

Il est tout d'abord possible d'accélérer le cycle biologique de la matière organique qui s'accumule dans les horizons supérieurs des sols acides (mor et moder) par un chaulage qui permet une remise en activité des micro-organismes du sol (Mai, Fiedler, 1972 ; Laatsch, 1964). Une telle opération a un effet relativement temporaire (quelques dizaines d'années) et ne permet pas d'augmenter le stock d'azote total. II est aussi possible d'intervenir par fertilisation minérale ou organique.

Ce type d'apport, s'il est efficace, a cependant un certain nombre d'inconvénients. II est coûteux et a un effet limité dans le temps (quelques années). De plus, il entraîne des pertes importantes d'azote par lessivage en raison du caractère brutal de cette intervention.

Une troisième possibilité est l'utilisation des propriétés de certaines espèces qui fixent l'azote atmosphérique $\left(\mathrm{N}_{2}\right)$ et le transfèrent à la plante-hôte sous forme d'azote combiné $\left(\mathrm{NH}_{4}\right)$.

Par le jeu du cycle biologique (retombée des feuilles, excrétion racinaire, décomposition de la biomasse racinaire), l'ensemble de l'écosystème forestier est enrichi en azote (Tarrant, Trappe, 1971). Diverses espèces utilisables en forêts tempérées sont susceptibles de fixer l'azote de l'air. 
En dehors des espéces herbacées et arbustives dont nous ne parlerons pas ici, il existe deux grands types d'espèces arborescentes pouvant fixer l'azote de l'air :

- les Légumineuses associées à des Rhizobia;

etc...).

- diverses espèces associées à des Actinomycètes du genre Frankia (Aulne, Casuarina,

Dans cet article, nous nous intéresserons uniquement aux Légumineuses arborescentes.

\section{DE L'INTÉRÊT DE L'UTILISATION DES LÉGUMINEUSES ARBORESCENTES}

Nous pouvons distinguer trois utilisations potentielles de telles essences en forêt :

- en association avec d'autres essences non fixatrices,

- en plein pour la production de biomasse,

- en plein pour la production de bois d'œuvre.

\section{Utilisation en association avec d'autres essences}

Les peuplements ainsi mélangés pourraient présenter divers avantages. Certaines Légumineuses fixent l'azote de l'atmosphère grâce à des Rhizobia (bactéries gram-négatif) associés, dans des nodules aux racines de la plante-hôte. II existe le plus souvent une spécificité plante-hôte/ Rhizobium.

Il est donc souvent nécessaire d'introduire à la fois le Rhizobium et la Légumineuse exotiques.

"Tenter d'acclimater une Légumineuse étrangère à la région sans s'être préoccupé de son associè habituel pourrait, même en dépit de la préexistence d'autres espèces de Légumineuses, indigènes celles-là, réserver quelques déconvenues "(Boullard, 1967).

En apportant l'azote par cette voie, le forestier pourrait ainsi accélérer la croissance des essences principales associèes à l'espèce fixatrice, surtout si ces espèces sont exigeantes en azote comme les Frênes, les Érables ou les Noyers.

Le Robinier (Robinia pseudacacia) fixe entre 100 et $300 \mathrm{~kg} / \mathrm{ha} / \mathrm{an}$ d'azote, alors que les microorganismes libres du sol n'en fixent que 2 à $5 \mathrm{~kg} / \mathrm{ha} / \mathrm{an}$. Notons que les précipitations (surtout en zones polluées) peuvent assurer des apports non négligeables au sol (5 à $15 \mathrm{~kg} / \mathrm{ha} / \mathrm{an}$ et parfois plus).

Les peuplements purs posent de nombreux problèmes d'ordre sanitaire.

La simplification des écosystèmes forestiers en particulier dans les peuplements artificiels diminue l'aptitude globale à absorber les chocs; elle est génératrice à long terme d'instabilité et de fragilisation. On peut penser que l'installation des peuplements mélangés à base de Légumineuses arborescentes pourrait apporter une moins grande fragilité.

La croissance des espèces fixatrices d'azote est en général très rapide dans les premières années d'installation. La mise en place d'un tel peuplement de remplissage pourrait améliorer l'élagage et la forme de l'espèce principale. Inversement la conduite de tels peuplements mixtes pourrait poser des problèmes de sylviculture difficile. II faut en effet que l'espèce fixatrice d'azote reste subordonnée à l'espèce principale productrice de bois d'œuvre. Il faut enfin que le coût de telles plantations ne soit pas prohibitif et que les avantages ne soient pas annihilés par les inconvénients. 


\section{Utilisation en plein pour la production de biomasse}

L'INRA et I'AFOCEL ont commencè des recherches sur les taillis à courte révolution permettant de produire des quantités importantes de matière première homogène pour les industries de trituration, pâtes et panneaux et pour éventuellement la production de méthanol.

C'est essentiellement l'Aulne qui a été utilisè dans ces essais (Teissier du Cros, 1987).

Cependant des expérimentations ont été entreprises dans le Sud de la France avec des

Légumineuses: Robinia pseudacacia, Gleditschia triacanthos, Sophora japonica.

Bien que les deux dernières espèces n'aient pas donné de résultats vraiment probants, il serait nécessaire de se pencher sur le problème de choix des provenances. Comme nous allons le voir, dans la partie descriptive des essences qu'il serait possible d'introduire, les aires naturelles de répartition sont parfois très étendues et couvrent donc des conditions écologiques très variées.

Pour Robinia pseudacacia, I'AFOCEL (Bonduelle et al., 1984) cite le chiffre de $3,8 \mathrm{~m}^{3} / \mathrm{ha} /$ an sans prendre en compte le volume des branches. Ces résultats sont loin des chiffres publiés par les Hongrois et les Roumains.

\section{Utilisation en plein pour la production des bois d'œuvre}

Certaines Légumineuses pourraient produire un bois de qualité. Si l'on se réfère aux dimensions de certaines espèces dans leur pays d'origine, on peut espèrer une bonne productivité en France à condition de rechercher les meilleures provenances possibles.

Gleditschia triacanthos atteint couramment 20 à $25 \mathrm{~m}$ dans la forêt américaine pour un diamètre de 0,60 à $0,90 \mathrm{~m}$; la hauteur maximale atteinte pour cet arbre est de $48 \mathrm{~m}$ pour $1,80 \mathrm{~m}$ de diamètre.

Sophora japonica atteint 20 à $27 \mathrm{~m}$ en Chine pour 230 à $400 \mathrm{~cm}$ de circonférence.

Dans leur aire d'origine ces essences peuvent être utilisées pour des usages nobles: èbènisterie et menuiserie.

\section{DESCRIPTION DES LÉGUMINEUSES}

\section{SUSCEPTIBLES D'ÉTRE UTILISÉES EN FORÊT TEMPÉRÉE}

Cette liste n'est pas limitative. Les critères de choix ont èté les suivants:

- dimension potentielle,

- résistance aux basses températures (existence dans les parcs et jardins botaniques),

- possibilité de production de bois d'œurre.

Pour le moment, nous avons relevé 22 espèces possibles dont 7 nord-américaines, 12 en provenance d'Extrême-Orient, 1 en provenance du Proche-Orient et 2 hybrides d'origine horticole.

Nous ne décrirons que les espèces de la tamille des Fabacèes, qui seules sont susceptibles de noduler.

Seul, le Robinier taux-acacia (Robinia pseudo-acacia) ne sera pas ètudié ici, car largement dècrit dans d'autres ouvrages et articles. 


\section{F. VERNIER - F. LE TACON}

\section{Les espèces nord-américaines}

\section{- Cladrastis lutea K. Koch}

Famille des Fabacées (Papilionacées)

Virgilier à bois jaune

Yellow-wood

C'est un petit arbre de 10 à $15 \mathrm{~m}$ de haut (rarement 20). Ses feuilles composées imparipennées de 20 à $30 \mathrm{~cm}$ de longueur sont constituées de 5 à 11 folioles obovées de 8 à $10 \mathrm{~cm}$ de long sur 2,5 à $5 \mathrm{~cm}$ de large prenant une belle teinte jaune à l'automne. Les fleurs blanches sont en panicules terminales de $36 \mathrm{~cm}$ maximum. Les gousses plates de 5 à $10 \mathrm{~cm}$ de long contiennent 4 à 6 graines.

Aire de repartition: Est des USA, de la Virginie à l'Arkansas et de la Caroline du Nord au Kentucky et au Tennessee.

Écologie: il aime les sols frais et profonds moyennement calcaires. II est résistant au froid.

Comportement en France : cette essence a résisté à des températures de $-34^{\circ}$ à $-35^{\circ} \mathrm{C}$ au Jardin botanique de Samoens (73) (Farille, communication orale). II existe deux sujets à l'arboretum des Barres, âgés de plus de 50 ans ayant $13 \mathrm{~m}$ de haut, et $128 \mathrm{~cm}$ et $72 \mathrm{~cm}$ de circonférence à hauteur d'homme (Durand, communication orale).

Utilisation : c'est un bois à aubier blanc et duramen jaune veiné, lourd, assez nerveux.

\section{- Robinia luxurians (Dieck) Schneid.}

Famille des Fabacées (Papilionacées)

C'est un arbre de 12 à $15 \mathrm{~m}$ de haut, à feuilles composées pennées à 15-25 folioles, ovales, de 2,5 à $4 \mathrm{~cm}$ de longueur sur 1 à $2 \mathrm{~cm}$ de largeur terminées par un cil raide. Ses fleurs sont rosepâle, en grappes compactes, dont le pédoncule est couvert de poils hérissés bruns. Les fruits sont des gousses de 7 à $10 \mathrm{~cm}$ de longueur à poils glanduleux et raides.

Aire de répartition: Colorado, Utah, Nouveau-Mexique, Arizona.

Écologie : il résiste à des froids de $-23^{\circ}$ à $-29^{\circ} \mathrm{C}$.

Comportement en France : un sujet existe au Jardin botanique de Besançon, âgé de 28 ans, il mesure une douzaine de mètres (Millet, communication orale).

Utilisation : non connue.

\section{- Robinia viscosa Vent.}

Famille des Fabacées (Papilionacées)

C'est un arbre d'une douzaine de mètres de haut et de 0,90 à $1 \mathrm{~m}$ de circonférence. Ses feuilles composées pennées mesurent 18 à $20 \mathrm{~cm}$ et sont constituées de 13 à 21 folioles de 4 à $5 \mathrm{~cm}$ de long sur $2 \mathrm{~cm}$ de large, pubescentes en dessous. Les gousses mesurent 5 à $9 \mathrm{~cm}$ de long et contiennent plusieurs graines marbrèes brun-rouge. Les rameaux sont parsemés d'èpines.

Aire de répartition: du Sud-Est des États-Unis à la Caroline du Nord et à l'Alabama.

Écologie : il résiste à des températures minimales de $-29^{\circ}$ à $-37^{\circ} \mathrm{C}$.

Comportement en France : il existe trois sujets aux Barres qui mesurent $8 \mathrm{~m}$ de haut pour $47 \mathrm{~cm}$, $38 \mathrm{~cm}$ et $23 \mathrm{~cm}$ de circonférence. Ils sont âgés de 35 ans (Durand, communication orale).

Utilisation : non connue. 


\section{- Cladrastis sikokiana (Makino) Makino}

Famille des Fabacées

C'est un arbre de $20 \mathrm{~m}$ de haut pour $70 \mathrm{~cm}$ de diamètre. Ses feuilles caduques sont alternes composées imparipennées de 15 à $30 \mathrm{~cm}$ de long avec 9 à 11 feuilles. Ses fleurs sont blanches, ont 15 à $20 \mathrm{~mm}$ de long en panicules terminales courtement pubescentes. Les gousses mûrissent en automne, elles sont plates, linéaires. Elles mesurent 5 à $10 \mathrm{~cm}$ de long et contiennent 3 à 10 graines.

Aire de répartition: endémique au Japon central et occidental, Honshu, Shikoku, Kyushu.

Écologie : identique à Cladrastis platycarpa.

Comportement en France : non connu.

Utilisation : il possède un bois jaune à porosité diffuse, utilisé dans la construction, la tournerie.
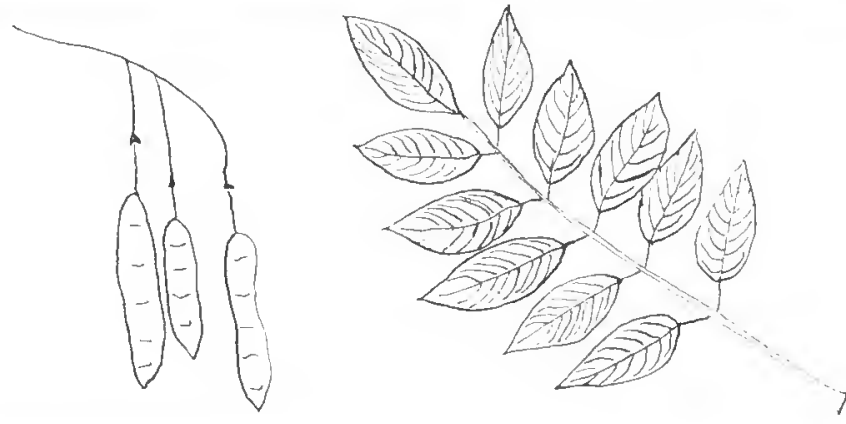

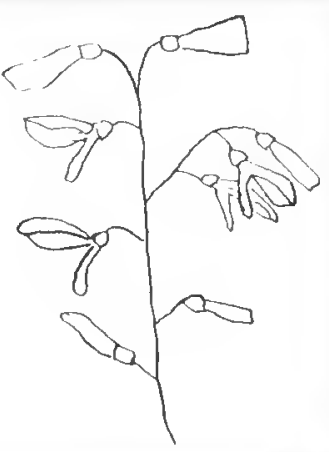

Cladrastis sikokiana

\section{Cladrastis sinensis Hemsl.}

Famille des Fabacées

Virgilier de Chine

C'est un arbre de 15 à $25 \mathrm{~m}$ de haut. Ses feuilles sont composées de 11 à 13 folioles étroites, oblongues, acuminées, de 7 à $12 \mathrm{~cm}$ de longueur. Elles sont glabres sur la face supérieure et glauques sur la face inférieure avec une pubescence rousse le long des nervures, les pétioles sont duveteux. Ses fleurs sont odorantes, blanc-rosé; elles ont une longueur de $12 \mathrm{~mm}$ et sont réunies en larges panicules terminales érigées de $25 \mathrm{~cm}$ de longueur et $15 \mathrm{~cm}$ de largeur. Les gousses mesurent 5 à $8 \mathrm{~cm}$ de longueur.

Aire de répartition: Ouest et Centre de la Chine.

Écologie : il aime les sols frais, fertiles même un peu calcaires. Il est un peu sensible au froid dans sa jeunesse; adulte, il résiste à des températures de $-20^{\circ}$ à $-23^{\circ} \mathrm{C}$. C'est une essence de pleine lumière.

Comportement en France : un sujet multicaule existe aux Barres, les circonférences des brins varient de 73 à $121 \mathrm{~cm}$ et la hauteur est de $12 \mathrm{~m}$, cet individu est du début du siècle.

Utilisation : non connue. 
Fleurs de Cladrastis sinensis, arboretum des Barres (Loiret)

Photo M. ANGEREAU

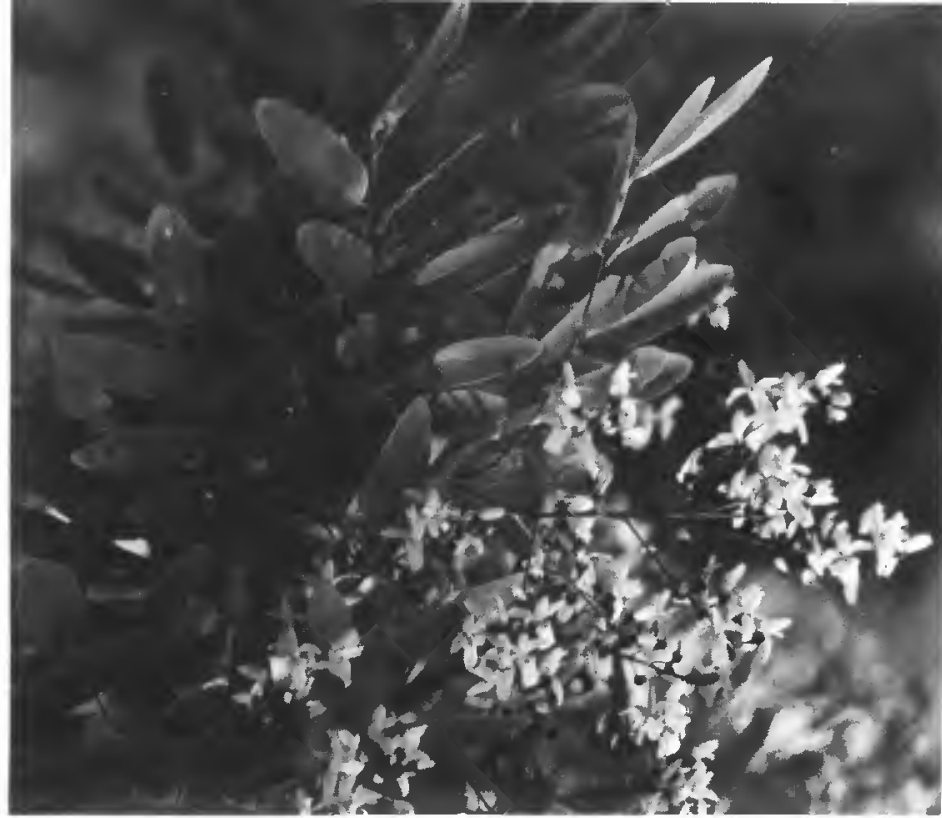

- Maackia amurensis (Rupr. et Max.) K. Koch

Famille des Fabacèes

Maackia de l'Amour

C'est un arbre de 20 à $25 \mathrm{~m}$ de haut à feuilles alternes, composées imparipennées constituées de 7 à 11 folioles elliptiques à ovales, à pointe courte au sommet et arrondies à la base; elles sont lisses, vert-foncé sur la face supérieure et plus pâle sur la face inférieure. Les fleurs sont dressées en panicules de 10 à $20 \mathrm{~cm}$ de haut. Les fruits sont des gousses de 3,5 à $5 \mathrm{~cm}$ de long.

Aire de répartition: vallée de l'Oussouri (affluent de l'Amour), Mandchourie.

Écologie: il préfère les sols argilo-siliceux, frais, nutritifs. Il résiste à des températures de $-29^{\circ} \mathrm{C}$.

Comportement en France: il existe une varièté, présente aux Barres, Maackia amurensis var buergerii qui a pour dimension $86 \mathrm{~cm}$ de circonférence pour $8 \mathrm{~m}$ de haut. II est àgé de plus de 40 ans mais pousse dans des conditions de sols difficiles. L'espèce-type ne mesure que $45 \mathrm{~cm}$ de circonférence et $7 \mathrm{~m}$ de haut et croît dans les mêmes conditions écologiques (Durand, communication orale).

Utilisation : c'est un arbre à croissance lente, à bois brun-jaunâtre, dur, tenace. Utilisè pour la construction et la menuiserie.

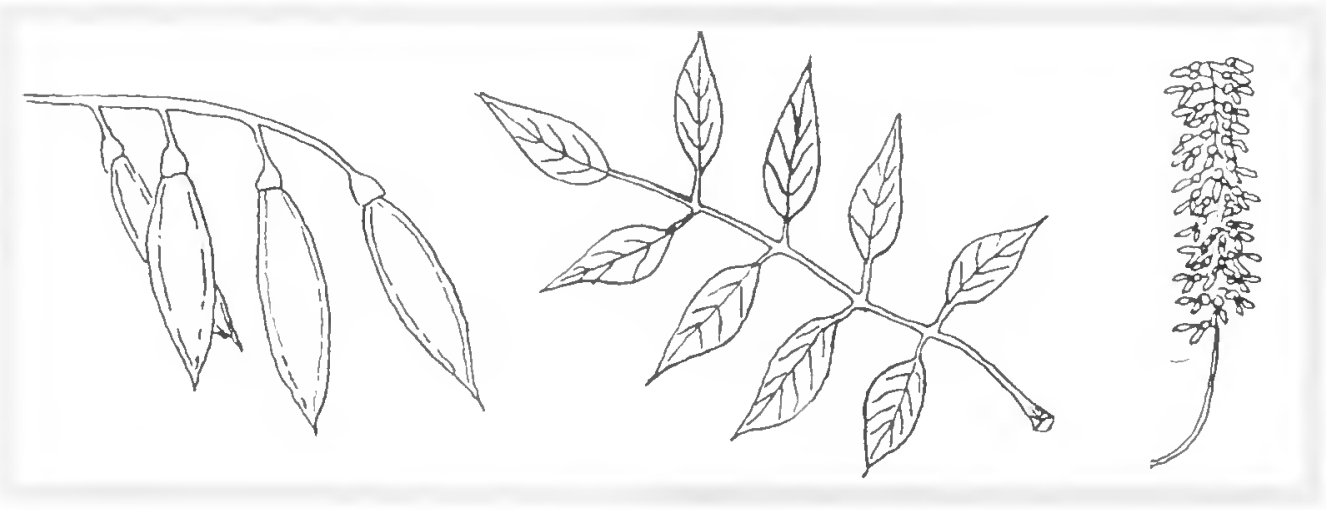


- Maackia chinensis Takeda

Famille des Fabacées

\section{Maackia de Chine}

Cet arbre peut atteindre $23 \mathrm{~m}$ de haut pour un diamètre de $35 \mathrm{~cm}$. Des feuilles composées de 11 à 13 folioles obtuses sont pubescentes sur la face inférieure. Les fleurs sont groupées en grandes panicules de 15 à $20 \mathrm{~cm}$ de long pour 10 à $12 \mathrm{~cm}$ de large. Elles sont de couleur blanche.

Aire de répartition: Hou-Peh, Centre de la Chine, Seu-Tchouan.

Écologie: cette essence n'a apparemment aucune exigence particulière en ce qui concerne l'exposition et le sol. Elle résiste à des températures de $-20^{\circ}$ à $-23^{\circ} \mathrm{C}$.

Comportement en France : deux individus se trouvent à l'arboretum des Barres, âgés de 60 ans et croissant dans des conditions de sols difficiles, ils atteignent $11 \mathrm{~m}$ de haut pour 39 et $55 \mathrm{~cm}$ de circonférence.

Technologie : non connue.

\section{- Sophora japonica L.}

Famille des Fabacées

Sophora du Japon Japan Pagoda-tree

C'est un arbre de 20 à $27 \mathrm{~m}$ de haut pour $2,50 \mathrm{~m}$ à $4 \mathrm{~m}$ de circonférence. Ses feuilles pennées de 20 à $25 \mathrm{~cm}$ de longueur sont composées de 7 à 17 folioles ovales oblongues de 3 à $5 \mathrm{~cm}$ de longueur, aiguës au sommet, vert-foncé, brillant dessus, glauques et légèrement pubescentes en dessous. Ses fleurs sont blanc-jaunâtre, mesurent de 1 à $1,5 \mathrm{~cm}$ de longueur et sont réunies en grandes panicules terminales lâches, dressées, jusqu'à $25 \mathrm{~cm}$ de longueur. Ses gousses sont longues de 5 à $8 \mathrm{~cm}$, pulpeuses et présentent la particularité d'être étranglées entre les graines.

Aire de répartition: Chine, Corée, largement introduit au Japon.

Écologie : cette essence préfère les sols suffisamment profonds, argileux, fertiles et légèrement calcaires, ni trop compacts, ni trop secs. Il supporte des températures de $-23^{\circ}$ à $-29^{\circ} \mathrm{C}$. II aime les expositions bien ensoleillées. Il n'est pas gêné par la pollution.

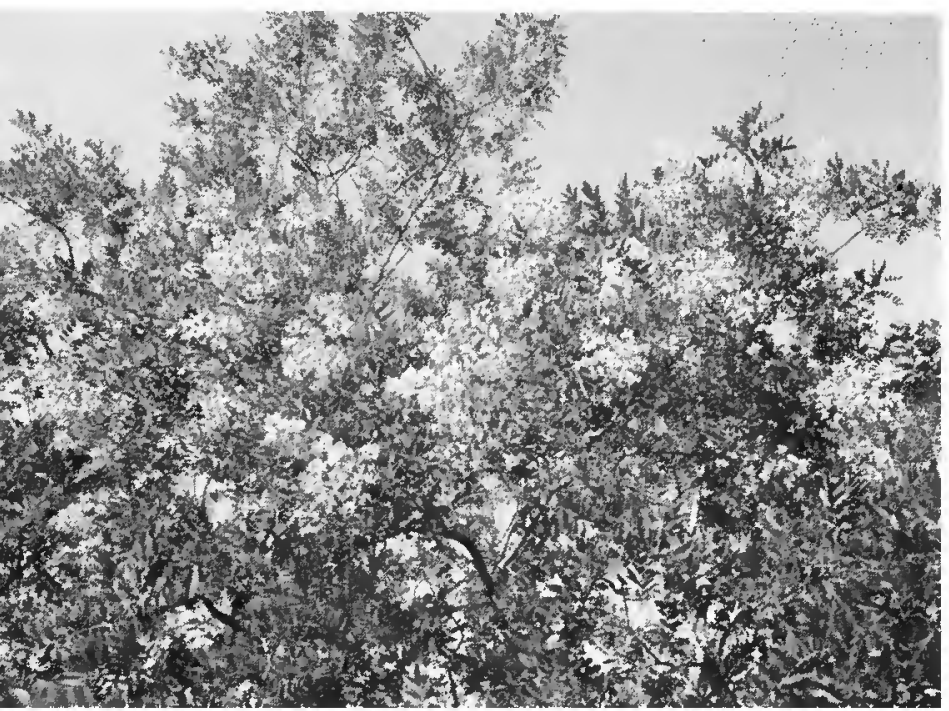

Comportement en France: cette espèce est présente dans de nombreux parcs et jardins de France. Nous en avons mesuré à Nancy un exemplaire de $16 \mathrm{~m}$ de haut et $199 \mathrm{~cm}$ de tour. À Metz, un individu atteint $3,80 \mathrm{~m}$ de circonfèrence pour $17 \mathrm{~m}$ de haut. II a produit des semis naturels dans notre pays (Pardé, 1943). Sophora japonica a été introduit en France en 1747 par le Père d'Incarville qui a envoyé des graines de Chine à Bernard de Jussieu. Elles ont été semées dans le jardin de Louis XV à Trianon (Guerrapain, 1809).

Fleurs de Sophora japonica, arboretum des Barres (Loiret) Photo M. ANGEREAU 
Utilisation : son bois a un aubier blanc et un duramen gris-jaunâtre ou roussâtre. II se travailie bien mais est un peu cassant et peu souple. II convient à l'ébénisterie et n'est pas exposé à la vermoulure. II existe une sous-espèce Sophora japonica ssp. pubescens (Tausch) Bosse.

\section{APTITUDE À LA NODULATION}

Toutes les Légumineuses ne fixent pas l'azote atmosphérique. Certaines espèces ou familles ne nodulent pas. D'autres espèces ayant la possibilité de noduler peuvent ne pas former de nodules si les Rhizobia spécifiques ne sont pas présents dans le sol d'introduction.

D'après la littérature, les Césalpinacées arborescentes ne nodulent pas dans leur aire d'origine.

Appartiennent aux Césalpinacées les genres suivants: Cercis, Gleditsia, Gymnocladus.

Ces genres ne peuvent donc être utilisés dans le but d'améliorer la disponibilité en azote des sols forestiers tempérés. Ils pourraient être utilisés dans l'optique de production de bois d'œuvre. Les Gleditsia en particulier seraient susceptibles de produire du bois de qualitè et atteignent des dimensions importantes dans leur aire d'origine aussi bien en Asie qu'en Amérique du Nord.

Seules seraient donc susceptibles d'être utilisées en forêt pour la fixation d'azote certaines Papilionacées (appelées maintenant Fabacées): Cladrastis, Maackia, Sophora, Robinia, qui ont été décrites au chapitre précédent (p. 216 et suivantes).

Le genre Robinia nodule systématiquement aussi bien dans son aire d'origine (Amérique du Nord) que dans toutes les conditions écologiques où il a été introduit en Europe.

Les Sophora nodulent systématiquement dans leur aire d'origine (Chine, Corée). Nous avons effectué des observations dans divers jardins botaniques ou parcs français. Nous n'avons jamais trouvé de nodules. II semble donc que les Sophora s'associent avec des Rhizobia spécifiques. Nous avons obtenu des souches isolées de Sophora chrysophylla et nous allons tenter de les introduire au moins dans les conditions de laboratoire.

La situation symbiotique de Maackia est mal définie. D'après Brewbaker, Maackia amurensis fixe l'azote alors que Maackia chinensis ne serait pas nodulant.

Certaines observations non publiées laisseraient cependant supposer que cette espèce nodulerait dans son aire d'origine.

La situation est également confuse pour le genre Cladrastis. Cladrastis platycarpa (Japon) ne nodulerait pas (Brewbaker, 1984 ; Dommergues, communication orale).

Cladrastis lutea (Amérique du Nord) pourrait être nodulé dans son aire d'origine.

Nous n'avons aucune information concernant Cladrastis sikokiana.

\section{CONCLUSIONS}

En dehors des espèces du genre Robinia, la Légumineuse arborescente qui pourrait ètre la plus intéressante pour améliorer la fixation biologique de l'azote en forêt tempérée à hiver rigoureux serait Sophora japonica. 


\section{F. VERNIER - F. LE TACON}

Malgrè l'absence, en France, de Rhizobium spécifique, cet arbre atteint dans divers parcs et jardins des dimensions importantes. À Metz et à Nancy, il atteint isolè un peu moins de $20 \mathrm{~m}$ de hauteur avec des diamètres importants (63 cm à Nancy, $120 \mathrm{~cm}$ à Metz).

Des essais d'introduction de Rhizobia spécifiques devraient permettre dans les prochaines années de déterminer ses capacités de fixation symbiotique de l'azote de l'air.

Des espèces des genres Cladrastis et Maackia seraient également susceptibles d'être utilisées pour améliorer la disponibilité en azote des sols forestiers en forêt tempérée. Cependant leur capacité à noduler est encore très mal connue, même dans leur aire d'origine.

Les espèces des genres Cercis, Gleditsia et Gymnocladus ne peuvent convenir à cet usage en raison de leur incapacité à noduler (famille des Césalpinacées).

En dehors de l'aspect fixation d'azote, les Gleditsia pourraient être introduits pour la production de bois d'œuvre.

Gleditschia triacanthos a un comportement tout à fait satisfaisant dans l'Est de la France (20 m de haut pour 40 à $50 \mathrm{~cm}$ de diamètre).

Cette espèce a la particularité de porter des épines redoutables. II existe cependant des variétés inermes.
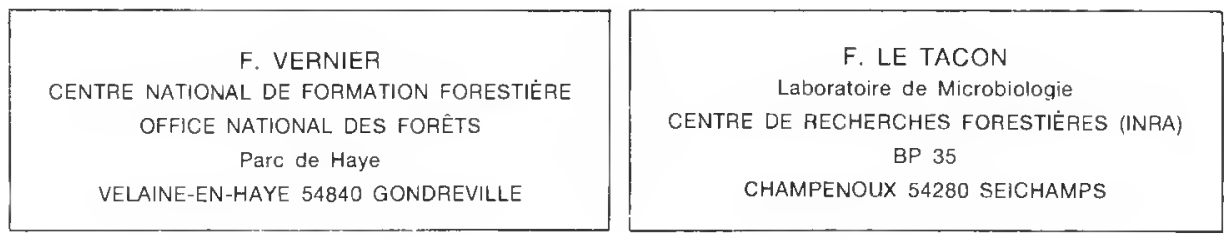

\section{BIBLIOGRAPHIE}

BONNEAU (M.). - La fertilisation forestière. In : Écologie forestière / P. Pesson Ed. - Paris : Gauthier-Villars, 1974. - pp. 99-113.

BONDUELLE (P.), BOULAY (M.), CAUVIN (B.), CHAPERON (H.), DELEPORTE (P.), DESTREMAU (F.X.), MARIEN (J.N.), MARQUESTAUT (J.), TOUZET (G.), VAUDELET (-). - Culture de biomasse ligneuse, taillis à courte rèvolution. - Paris: AFOCEL, 1982. - pp. 22-24-27.

BOSSARD (R.), CUISANCE (P.). - Arbres et arbustes d'ornement des régions tempèrèes et mèditerranéennes. - Paris: Lavoisier, 1984. - pp. 275-278-284.

BOULLARD (B.). - Essenzes "nobles" et espèces fixatrices d'azote libre ou l'incitation à la solidarité en sylviculture. - La forêt privée, $\mathrm{n}^{\circ} 158,1984$, pp. 19-26.

BOULLARD (B.). - Vie intense et cachèe du sol, essai de pédobiologie vègètale. - Paris : Flammarion, 1967. - Pp. 157-185.

BREWBAKER (J.L.), HALLIDAY (J.). - Nitrogene, fixing tree research reports. - Hawaii: NFTA, 1984. DP. 29-30-39-45.

BROCKMAN (-), ZIM (-), MERRIBES (-). - Guide des arbres de l'Amèrique du Nord. - Paris: M. Broquet, 1982. - pp. 186-192.

DOMMERGUES (Y.). - Communications orales.

DURAND (R.). - L'arboretum national des Barres. - Nogent-sur-Vernisson: ENITEF, 1984. - p. 64.

FARILLE (-). - Communication orale.

FOWELLS (H.A.). - Sylvics of forests trees of the U.S. - Forest Service US - Department of Agriculture, 1965. - pP. 198-201.

GUERRAPAIN (M.T.). - Notice sur la culture du Sophora, du Platane et de l'Aulne. - Paris: D. Colas, 1809.

HAUSSER (K.). - Ergebnisse von Düngungsversuchen zu 50 bis 70 jahringen Fichtenbeständen auf oberem Buntsandstein des Württemb. Schwarzwalds. - Allgemeine Forst-und Jagdzeitung, ${ }^{\circ}{ }^{132,1961,}$ pp. 269291. 
KURATA (S.), - Illustrated important forest trees of Japan. - Japan forest technical Association, 1971. p. 152 .

LAATSCH (W.). - Der Aufbau fruchtbarer Waldböden. - Mitteilungen aus der Staatsforstverwaltung Bayern, 34, 1964, pp. 274-286.

MAI (H.). FIEDLER (H.J.). - Bodenmikrobiologische Untersuchungen an einem Düngungsversuch zu Fichtenrohhumus in Thüringerwald. - Zentralblatt für Bakteriologie, Parasitenkunden, infektionskrankheiten und Hygiene, val. 127, n6, 1972, pp. 618-631.

MILLET (-). - Communication orale.

MOIROUD (A.), CAPELLANO (A.). - Le Robinier Robinia pseudoacacia L., une espèce fixatrice d'azote interessante? - Annales des Sciences forestières, vol. 39, $n^{\circ} 4,1982$, pp. 407-416.

NYS (C.). - Fertilisation des peuplements adultes d'Épicéa commun dans le Massif Central. - Revue forestière française, vol. XXXVI, $\cap^{\circ} 4,1984, \mathrm{pp} .313-318$.

PARDÉ (L.). - Les feuillus. - Paris: La Maison Rustique, 1943. - pp. 221-234.

TARRANT (R.F.), TRAPPE (J.M.). - The role of Alnus in improving the forest environment. - Plant and Soil, special volume, 1971 , pp. 335-348.

TEISSIER DU CROS (E.). - Production and improvment of productivity of forest biomass in the European Community. - Florida meeting energy from biomass, 1987.

VETVICKA (V.), MATONSOVA (V.). - Arbres et arbustes. - Prague : Artia ; Paris: Grund, 1984. - pp. $242-$ 249.

VIRO (P.J.). - Economics of fertilisation in private forestry. - $3^{e}$ conférence internationale de production et fertilisation forestière, 1969.

\section{PUBLICATIONS DE L'ARMEF}

L'ARMEF met au point et diffuse des notices, dépliants et ouvrages, ayant trait à l'exploitation torestière et la mécanisation qui méritent d'être mieux connus. En voici une liste:

Notices:

- Pour un abattage sans accident;

- Sécurité du travail dans l'exploitation torestière ;

- Ébranchage à la scie à chaîne :

- Manutention avec la grue hydraulique à grappin :

- Grue à longue portée en éclaircie ;

- Scarification.

Plaquettes :

- Méthode d'exploitation des bois en courtes et grandes longueurs;

- Méthode d'exploitation d'une coupe de taillis ;

- Méthode d'exploitation semi-mécanisée des éclaircies en ligne de résineux;

- Méthode d'exploitation mécanisée des éclaircies ;

- Méthode d'exploitation semi-mécanisée des chablis ' ;

- La scie à chaîne : entretien - rodage ;

- Pince de débardage à trois bras;

- La débroussailleuse : entretien - rodage;

-_ Sécurité : attention au feu ;

- Sécurité dans le bûcheronnage des chablis ${ }^{*}$;

- La débroussailleuse : sécurité.

(' Nous attirons spécialement l'attention sur l'intérêt de ces deux dépliants).

\section{Ouvrages:}

- Catalogue des machines d'exploitation forestière - $3^{e}$ édition 1986 (avec mise à jour 1988 des adresses et téléphones des constructeurs et distributeurs).

— Équipements d'exploitation forestière - $1^{\text {re }}$ édition 1984 (avec mise à jour 1988 des adresses et téléphones des constructeurs et distributeurs).

- Le travail à la scie à chaîne.

- Le tracteur agricole pour le transport des bois.

Pour toute commande, s'adresser à l'ARMEF (85, rue Lafayette - 75009 PARIS - tél. (1) 42806977 ). 